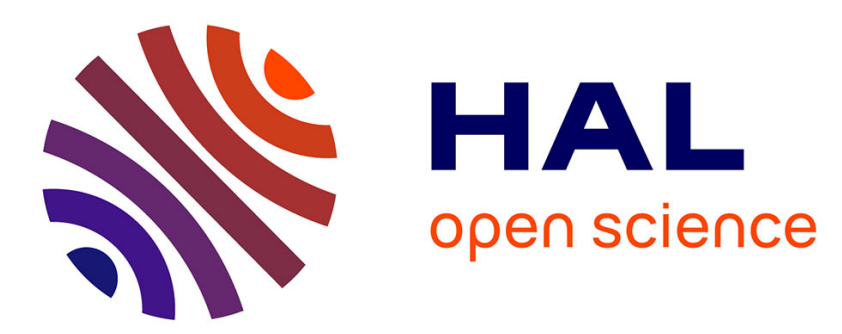

\title{
Du réparateur au facilitateur : changement de regard sur l'accompagnement
}

Christophe Schmitt, Julien Husson

\section{To cite this version:}

Christophe Schmitt, Julien Husson. Du réparateur au facilitateur: changement de regard sur l'accompagnement. Entreprendre \& Innover, 2014, 2014/2-3 (21-22), pp.10-18. 10.3917/entin.021.0010 . hal-02989699

\section{HAL Id: hal-02989699 \\ https://hal.science/hal-02989699}

Submitted on 11 Nov 2020

HAL is a multi-disciplinary open access archive for the deposit and dissemination of scientific research documents, whether they are published or not. The documents may come from teaching and research institutions in France or abroad, or from public or private research centers.
L'archive ouverte pluridisciplinaire HAL, est destinée au dépôt et à la diffusion de documents scientifiques de niveau recherche, publiés ou non, émanant des établissements d'enseignement et de recherche français ou étrangers, des laboratoires publics ou privés. 


\title{
Du réparateur au facilitateur : changement de regard sur l'accompagnement entrepreneurial
}

\author{
Christophe SCHMITT \\ Titulaire de la Chaire « Entreprendre » \\ christophe.schmitt@univ-lorraine.fr \\ Université de Lorraine \\ Julien HUSSON \\ Maître de Conférences, HDR, Gestion \\ Université de Lorraine \\ julien.husson@univ-lorraine.fr
}

Christophe SCHMITT : Titulaire de la Chaire «Entreprendre » à I'Université de Lorraine, ses réflexions portent sur les différentes dimensions du processus entrepreneurial dans une perspective essentiellement systémique. II a écrit différents ouvrages et articles dans le domaine. Son dernier ouvrage paru aux Editions Eyrolles s'intitule Réussir sa création d'entreprise sans business plan. II est actuellement professeur associé à la Louvain School of Management de Louvain-la- Neuve en Belgique.

Julien HUSSON : Directeur de l'IAE de Metz, ses travaux portent sur la conception de projets complexes dans les organisations. II a accompagné différentes organisations dans le développement de leur projet en mettant en œuvre des démarches innovantes. 


\section{Résumé :}

L'accompagnement a été souvent envisagé sous la posture du réparateur, c'est-àdire de l'expert qui amène l'entrepreneur à la création d'entreprise, en prenant notamment appui sur le plan d'affaires. Aujourd'hui émergent d'autres modalités d'accompagnement, pouvant être envisagées de façon complémentaire. Dans cet article, nous nous intéresserons principalement à une posture qui provient de l'accompagnement thérapeutique issu de la systémique : la posture du facilitateur. Point clés :

- il existe des postures complémentaires en matière d'accompagnement entrepreneurial : la posture classique du réparateur et la posture émergente du facilitateur ;

- la posture du facilitateur engendre de nouveaux mécanismes en matière d'accompagnement entrepreneurial : la problématisation et la construction d'un scénario ;

- la posture du facilitateur est à rapprocher de la posture maïeutique ;

- la posture du facilitateur amène l'entrepreneur à construire son propre scénario qu'il est invité à confronter auprès de différentes parties prenantes.

Si l'entrepreneuriat se renouvelle fortement actuellement à travers des concepts comme le Lean Startup, le Design Thinking ${ }^{1}$ ou encore l'effectuation, il est nécessaire de s'interroger sur l'accompagnement entrepreneurial et, notamment, sur ses évolutions récentes. Dans cet article, il s'agit de renouveler le regard sur l'accompagnement entrepreneurial en introduisant tout un pan de littérature encore

\footnotetext{
${ }^{1}$ Dossier spécial du n¹9 de la Revue Entreprendre \& Innover.
} 
quasiment inexploré dans le domaine de l'entrepreneuriat : l'accompagnement systémique. Nous montrerons dans cet article que l'accompagnement entrepreneurial relève de deux postures : celle du réparateur et celle du facilitateur. Nous présenterons ces deux postures en insistant tout particulièrement sur les caractéristiques de chacune d'elles. Ensuite, en s'appuyant sur une expérience, étayée depuis plusieurs années par une démarche nommée IDéOC, nous illustrerons plus particulièrement la posture du facilitateur à travers le cas de Dominika, jeune entrepreneure dans le domaine de l'import-export d'outillage industriel. Nous y reviendrons plus particulièrement sur des mécanismes mis en évidence dans cette posture : la problématisation et la construction d'un scénario, comme sur les enjeux de cette posture dans l'accompagnement entrepreneurial : la conception et la traduction.

\section{Les différentes postures de l'accompagnement entrepreneurial}

En s'intéressant à l'accompagnement entrepreneurial, les travaux ont largement porté sur les mécanismes mis en œuvre et peu sur la nature de l'accompagnement proposé. II existe pourtant une littérature féconde en matière d'accompagnement et pourtant peu mobilisée dans le domaine de l'entrepreneuriat : l'accompagnement inscrit dans une perspective systémique, notamment à travers la mobilisation de thérapies systémiques ${ }^{2}$. II ressort de cette littérature en matière d'accompagnement la coexistence de deux postures d'accompagnement que nous proposons de mobiliser au niveau de l'accompagnement entrepreneurial. La première posture, celle qui est encore dominante actuellement et qui peut-être symbolisée par le plan

\footnotetext{
${ }^{2}$ La thérapie systémique se différencie de la plupart des autres thérapies, en ce sens qu'elle prend l'individu dans son environnement - dans un ensemble de systèmes - et a, de ce fait, une vision plus élargie qui aide le thérapeute dans le traitement de son patient (Caillé P., Un et un font trois, Paris, ESF, 1991).
} 
d'affaires, est celle du réparateur. La seconde, qui émerge depuis quelques temps, donne à voir une posture différente de l'accompagnement, pouvant être qualifiée de «facilitateur ». Quels sont les grands principes liés à ces deux postures d'accompagnement ? Pour répondre à cette question, nous nous sommes appuyés sur la métaphore des planètes Alpha et Beta proposée par Caillé ${ }^{3}$ :

- sur Alpha, chaque organisation est dotée d'un « engin » qui fournit automatiquement les réponses adéquates à tous les problèmes pouvant surgir. Lorsque cet engin est en panne, l'organisation fait appel à un « réparateur » qui en assurera les réglages nécessaires et remplacera les éléments défectueux ;

- sur Bêta, chaque organisation construit ses outils avec les moyens dont elle dispose. Ces organisations peuvent, en cas de difficulté, faire appel à un « facilitateur ». Celui-ci ne vient pas apporter la solution, il se sert avant tout de sa position pour aider le système à se donner une représentation réflexive ${ }^{4}$ de lui-même, à ne pas s'enfermer dans son point de vue, à percevoir autrement ce qui est peut l'être, à redevenir acteur et créateur de son devenir.

\section{La posture du réparateur dans l'accompagnement entrepreneurial}

De manière allégorique, la planète Alpha considère l'accompagnement comme la volonté d'arriver à un objectif : la création d'entreprise. L'accompagnateur apparaît comme l'expert qui le permet. A partir d'une situation donnée par l'entrepreneur, l'accompagnateur va favoriser la transformation en création d'entreprise sans forcément remettre en cause la situation donnée. L'accompagnateur apparaît ici

\footnotetext{
${ }^{3}$ Caillé P., Un et un font trois, Paris, ESF, 1991.

${ }^{4}$ Type de représentation permettant l'auto-référence : une solution n'est pas cherchée en dehors d'un système mais au-dedans de lui, au travers de la prise en compte simultanée de l'action et de l'auteur de l'action.
} 
comme un expert en création d'entreprise, pendant même que le plan d'affaires est considéré comme l'outil de la transformation en création d'entreprise. Il s'agit de la posture du réparateur. Elle renvoie essentiellement à des méthodes déductives, incarnées dans la résolution de problèmes. Cela se caractérise par quatre étapes: l'identification (comprendre la situation initiale), l'analyse (recherche des points qui posent problèmes et qui seraient à travailler), la solution (une ou des solutions à mettre en place) et la mise en œuvre (plan d'action). La place de l'action, qui arrive à la fin, est ici un élément majeur. La posture du réparateur amène à séparer observation et action, en donnant une part belle à l'observation ${ }^{5}$, laquelle se synthétise encore une fois autour du sacro-saint plan d'affaires.

\section{La posture du facilitateur dans l'accompagnement entrepreneurial}

De l'autre côté, la planète Bêta considère l'accompagnement comme une volonté de construire du sens, de revisiter une situation donnée, voire de proposer une alternative à la formulation d'un projet. La création d'entreprise n'est pas le but en soi, ce n'est qu'un passage, une orientation possible pour l'entrepreneur. Dans cette perspective, il ne s'agit plus de considérer l'entrepreneuriat comme une situation donnée d'emblée, mais bien comme une situation à construire. La figure emblématique de cette posture n'est pas le plan d'affaires mais l'opportunité d'affaires, c'est-à-dire l'intégration d'une idée dans un environnement. La création d'entreprise apparaît avant tout comme une forme particulière d'un projet. L'accompagnateur joue alors le rôle de « facilitateur », celui qui amène les porteurs de projet à se poser des questions par rapport à une situation initiale donnée, simplement affirmée. Les méthodes mobilisées par le facilitateur relèvent clairement

\footnotetext{
${ }^{5}$ Nous entendons par observation le fait de regarder son environnement et de ne pas agir sur lui. Une étude de marché par exemple rentre dans une logique d'observation. A contrario, nous entendons par action, le fait d'agir dans et/ou sur l'environnement.
} 
de la maïeutique : elles visent à amener l'entrepreneur à prendre conscience de ce qu'il sait implicitement, à l'exprimer et à l'évaluer. On passe de la logique de résolution de problème à celle de problématisation, c'est-à-dire à celle de construction du sens. Cela se concrétise par la formulation d'une situation souhaitée, celle d'état final par rapport à un état initial. On retrouve bien ici la logique systémique formulée par Watzlawick ${ }^{6}:$ «Ce n'est [...] le passé, mais le futur, qui détermine le présent », ou encore les travaux sur la conception de Simon ${ }^{7}$. Dans cette configuration, le plan d'affaires apparaît comme un outil parmi d'autres amenant l'entrepreneur à discuter la situation initiale et à projeter et formuler une situation finale à travers une opportunité d'affaires. Originellement, le facilitateur se distingue du réparateur dans la mesure où il n'apporte aucune solution clé (Couteret et Audet, 2008). La problématisation, à la mise en place de laquelle il contribue, se caractérise par deux étapes : amener l'entrepreneur à se poser des problèmes ${ }^{8}$ et à poser une problématique ; l'amener à identifier son action pour mieux l'appréhender et la maîtriser. Au bout de ce questionnement, le porteur de projet comprend mieux ce qu'il souhaite construire et voit apparaître la cohérence des éléments composant le projet entrepreneurial. L'image de la planète Bêta suggèrerait que l'entrepreneur, portant le projet, a en lui les éléments du scénario qu'il souhaiterait développer. II conviendrait donc de lui faire accoucher de ce scénario. L'objectif du facilitateur est de révéler au porteur de projet son propre modèle organisant pour l'aider dans l'action. Dans la posture du facilitateur, l'observation et l'action jouent un rôle

\footnotetext{
${ }^{6}$ Watzlawick P. (1988), Effet ou cause ? dans Watzlawick P. (coordination), L'invention de la réalité, contribution au constructivisme, Seuil, p. 73-78.

${ }^{7}$ Pour Simon est concepteur « quiconque imagine quelque disposition visant à changer une situation existante en une situation préférée ".

Simon H. A. (1991), The sciences of the artificial, Dunod.

${ }^{8}$ II convient de faire la différence entre question et problème. Nous entendons par question la demande que l'on adresse à quelqu'un en vue d'apprendre quelque chose de lui et par problème la difficulté liée à une situation finale par rapport à une situation initiale.
} 
prépondérant. Au lieu d'avoir une posture contemplative, l'entrepreneur est invité à lier observation et action. Car, c'est dans l'action qu'il est possible de répondre aux questions qu'il se pose au cours de l'observation. C'est aussi dans l'action que de nouvelles questions peuvent émerger, celles-ci-mêmes qui n'auraient pas pu voir le jour sans action. II s'agit bien de (ré)introduire l'action dans les démarches entrepreneuriales et, plus particulièrement, de sortir des seules postures contemplatives que l'on retrouve au niveau du plan d'affaires. Le facilitateur a notamment pour rôle d'aider les acteurs du système à se doter d'une représentation réflexive, en vue de trouver des solutions par eux-mêmes, en regard des actions à mener.

Tableau récapitulatif des deux postures de l'accompagnement entrepreneurial

\begin{tabular}{|c|c|c|}
\hline & Posture du réparateur & Posture du facilitateur \\
\hline $\begin{array}{l}\text { "Je souhaite créer une } \\
\text { entreprise " }\end{array}$ & Objectif à atteindre & $\begin{array}{ll}\text { Passage possible parmi } \\
\text { d'autres possibilités à } \\
\text { envisager }\end{array}$ \\
\hline Objectif & La création d'entreprise & La construction de sens \\
\hline $\begin{array}{l}\text { Rôle de } \\
\text { l'accompagnateur }\end{array}$ & $\begin{array}{l}\text { Faciliter la création } \\
\text { d'entreprise }\end{array}$ & $\begin{array}{l}\text { Faciliter la construction de } \\
\text { sens à partir d'une } \\
\text { situation finale par rapport } \\
\text { à une situation initiale }\end{array}$ \\
\hline Modalité d'approche & Résolution de problème & Problématisation \\
\hline Méthode & Déductive & Maïeutique \\
\hline Les étapes préconisées & $\begin{array}{l}4 \text { étapes : } \\
\text { - identification } \\
\text { - analyse } \\
\text { - solution } \\
\text {-mise en œuvre }\end{array}$ & $\begin{array}{l}2 \text { étapes : } \\
\text { - amener l'entrepreneur à } \\
\text { se poser des problèmes } \\
-\quad \text { identifier ce que } \\
\text { l'entrepreneur est en train } \\
\text { de faire }\end{array}$ \\
\hline $\begin{array}{l}\text { Lien entre observation et } \\
\text { action }\end{array}$ & $\begin{array}{l}\text { Prépondérance de } \\
\text { l'observation par rapport à } \\
\text { l'action }\end{array}$ & $\begin{array}{l}\text { Relation forte entre } \\
\text { l'observation et l'action }\end{array}$ \\
\hline Temporalité & $\begin{array}{l}\text { Le temps est linéaire allant } \\
\text { du présent au futur }\end{array}$ & $\begin{array}{l}\text { Le futur et le présent } \\
\text { interagissent de façon } \\
\text { récursive }\end{array}$ \\
\hline Place du plan d & Outil de référence & Un outil parmi d'autres \\
\hline Besoin d'information & $\begin{array}{l}\text { Par rapport au plan } \\
\text { d'affaires à remplir }\end{array}$ & $\begin{array}{l}\text { Par rapport à la nécessité } \\
\text { de construire du sens }\end{array}$ \\
\hline
\end{tabular}




\begin{tabular}{lll}
\hline La réussite & La faisabilité & La cohérence \\
Elément symbolique & Plan d'affaires & Opportunité \\
\hline Rapport aux autres & Logique concurrentielle & Logique partenariale \\
\hline
\end{tabular}

Les apports et les enjeux liés à la posture du facilitateur dans l'accompagnement entrepreneurial

Si l'on accepte le fait que l'accompagnement entrepreneurial peut avoir différentes postures, il est nécessaire de s'interroger plus particulièrement sur la posture qui est peu présente dans l'accompagnement de l'entrepreneuriat actuellement, celle du facilitateur, afin d'en comprendre les apports et les enjeux. Pour cela, nous nous appuierons sur notre expérience, étayée par la méthode IDéO@, notamment à travers le cas de Dominika, jeune entrepreneure souhaitant se lancer dans l'importexport. Les apports de la posture du facilitateur amènent à repenser l'entrepreneuriat et son accompagnement autour de nouvelles dimensions : la problématisation et la construction d'un scénario. Au niveau des enjeux, ils sont au nombre de deux : la capacité de concevoir ce futur et la traduction de cette conception. La capacité de concevoir est le levain de l'organisation des connaissances en projet entrepreneurial. Dans cette optique, il convient d'aider les porteurs de projet à concevoir leur scénario à partir de leur projet entrepreneurial. La traduction est, elle aussi, un enjeu car elle renvoie à la nécessité de coopération et de collaboration dans la plupart des projets. En effet, un projet n'est jamais le seul fait de l'entrepreneur; il implique des liens internes et externes. Néanmoins, encore faut-il que l'entrepreneur puisse traduire son projet entrepreneurial auprès de ses parties prenantes. 


\section{Les apports du facilitateur dans l'accompagnement entrepreneurial}

L'entrepreneur, comme le rappelle justement Filion ${ }^{9}$, a la possibilité de définir luimême l'espace dans lequel il souhaite évoluer. Il se retrouve dans une situation où il est nécessaire de problématiser le futur souhaité. La résolution de problème ne convient pas dans ce type de situation. En effet, les approches traditionnelles basées sur la résolution de problèmes sous-entendent que les éléments d'une situation sont donnés, alors que la situation est à construire. L'entrepreneur est amené à construire le scénario qu'il souhaite développer. Dans une logique de résolution de problème, le réparateur amène à intégrer un scénario à partir d'une situation donnée. Dans une posture de facilitateur, l'objectif est d'amener l'entrepreneur à construire son scénario à partir de différents scenarii possibles. Dans cette perspective, nous considérons, comme certains auteurs ${ }^{10}$ que l'opportunité d'affaires est avant tout un construit de l'entrepreneur. De plus, le scénario construit ne doit pas être considéré comme un optimum ni être considéré de façon figée. II est une construction de sens que se fait le porteur du projet de la situation dans laquelle il évolue. En cela, l'entrepreneuriat est proche de l'innovation, de la créativité ou de l'invention. Ces éléments sont typiques des logiques «projets », où la représentation du porteur de projet joue un rôle important. Celle-ci est amenée à évoluer dans le temps. L'entrepreneuriat, dans cette logique, s'apparente donc plus à un processus qu'à un résultat. Ces différents constats nous amènent à avancer que les difficultés rencontrées par les entrepreneurs sont plus liées à la construction de scénario qu'à la mise en place de

\footnotetext{
${ }^{9}$ Filion, L.-J. (1999), Tintin, Minville, l'entrepreneur et la potion magique, Les grandes conférences, Presses HEC

${ }^{10}$ Sur ce point voir notamment les travaux sur l'effectuation de Sarasvathy. Sarasvathy S. D. (2003), Entrepreneurship as a science of the artificial, Journal of Economic Psychology, Vol. 24, p. 203-220.
} 
solutions ${ }^{11}$. L'accompagnement évolue alors pour se porter plus sur le processus à piloter que sur le résultat à obtenir : la création d'entreprise. Dominika est une jeune entrepreneure. Le travail effectué avec elle, à travers la méthode IDéO@, a permis de l'aider à problématiser son idée de départ. Elle projetait de développer une activité d'import-export d'outillage pour l'industrie entre le Pologne et le France. Grâce à une question simple, «le projet, c'est quoi ? », Dominika a été amenée à envisager différentes dimensions de son projet, notamment sa définition même. II lui a été alors demandé d'exprimer son projet en une phrase unique. A partir de cette phrase, toute une série de questions a émergé et un dialogue s'est développé entre Dominika et le facilitateur, amenant à envisager différentes perspectives du projet. Celles-ci ont permis à leur tour la mise en évidence des dimensions oubliées (les entreprises de petite taille, par exemple), des dimensions peu compréhensibles (vouloir travailler en $B$ to $B$ et $B$ to $C$, en même temps, au début du projet), des dimensions paradoxales (comme un outillage de qualité $30 \%$ moins cher que l'outillage habituel) et des dimensions très claires (une qualité du produit indéniable). A partir d'une question de départ et d'une posture de facilitateur, un travail maïeutique s'est mis en place autour d'une séance d'une heure.

L'idée de problématisation amène le porteur de projet à construire un scénario, c'està-dire à se projeter. II s'agit pour les porteurs de projet d'imaginer des actions, leurs conséquences et leurs résultats sur leur environnement. Cette projection correspond à la représentation de la solution qu'il se propose de mettre en place pour répondre à la compréhension qu'il se fait de son environnement. Cette conscience se fait plus facilement lorsqu'il est aidé par un tiers pour arriver à se projeter dans un scénario plausible. L'accompagnateur n'agit donc pas comme un expert technique par rapport

\footnotetext{
${ }^{11}$ Schmitt C., Julien P.-A., Lachance R., Pour une lecture des problèmes complexes en PME : approche conceptuelle et expérimentation, dans Revue Internationale PME, 2002, vol 15, N², p. 35-62.
} 
à la création d'entreprise, mais comme un révélateur par rapport aux porteurs de projet. Bien que cela paraisse évidant à la lecture de cet article, il est important de souligner que ce mécanisme de projection, naturel par ailleurs, ainsi que notre capacité au quotidien à concevoir, sont rarement identifiés au niveau de l'accompagnement entrepreneurial. Si nous considérons que la posture du facilitateur est une dimension importante de l'accompagnement entrepreneurial, il est nécessaire d'y former les accompagnateurs. En effet, si certains acteurs de l'accompagnement vont dans ce sens, leur démarche s'avère bien souvent intuitive. II conviendrait de la caractériser et de la généraliser, afin de permettre aux accompagnateurs de pouvoir travailler sur la capacité de concevoir en entrepreneuriat.

La seconde séance avec Dominika a permis de construire le scénario qu'elle souhaitait à travers un ensemble de questions supplémentaires tirées de la méthode IDéO๑ : à la suite de la question« le projet, pour quoi ? »; « le projet fait quoi ? »; «le projet dans quel environnement ? »; «le projet dans quelle histoire ? ». Sans devoir détailler ici ladite méthode ${ }^{12}$, précisons toutefois que le scénario monté par Dominika a fait l'objet d'un travail de confrontation dans une posture de facilitateur. II s'agissait, notamment, d'aider Dominika à ouvrir des portes, pour découvrir ce qu'il y avait derrière elles. A la fin de la séance de travail, des portes ont été refermées par Dominika et d'autres sont restées ouvertes. Quelle que soit la situation, elle était capable de justifier ses choix et de montrer le cohérence de son scénario.

\section{Les enjeux liés à la notion de facilitateur}

\footnotetext{
${ }^{12}$ Schmitt C., IDéOC : une méthode pour aider l'entrepreneur à concevoir un scénario à partir d'une opportunité, dans Filion L.J., Ananou C., Schmitt C. (dir), Réussir sa création d'entreprise sans business plan, Editions Eyrolles, 2012, p. 117-129.
} 
En sus des apports du facilitateur, en l'occurrence la problématisation et la capacité de se projeter, il convient maintenant de mettre en relief les enjeux qui y sont. Pour cela, nous traiterons de façon successive la place de l'activité de conception et l'importance de l'activité de traduction au niveau de l'accompagnement entrepreneurial.

Nous entendons donc par «conception » la capacité des porteurs de projet à développer un dessein dans le but de réaliser un dessin ; c'est le scénario évoqué précédemment. Autrement dit, il s'agit de leur capacité de passer d'une idée (dessein) à une opportunité (dessin). Ainsi, est concepteur «quiconque imagine quelque disposition visant à changer une situation existante en une situation préférée ${ }^{13} »$. En transposant cette définition à notre problématique entrepreneuriale, nous pouvons considérer les entrepreneurs comme des concepteurs et l'accompagnement entrepreneurial comme une démarche aidant les entrepreneurs à concevoir. La conception demande un travail d'imagination organisé et structurant, où l'accompagnateur joue un rôle important permettant à l'entrepreneur de prendre conscience des éléments structurants de son projet. Cela requiert de la méthode, des efforts. Dans le cas de Dominika, le travail avec la méthode IDéO@ a permis la prise de conscience des éléments structurants de son projet : outillage, PME, qualité, pas de stock, confiance. Les premiers pas de Dominika sur le terrain l'ont amenée à confronter son scénario aux parties prenantes du projet. Ce travail de confrontation permet de tester la robustesse du projet et favorise les ajustements par rapport aux représentations des autres du scénario envisagé.

Concevoir un scénario par exemple, dans le cas de l'entrepreneur, n'est pas un acte isolé. II se fait en lien avec l'environnement du porteur de projet et en lien avec les

\footnotetext{
${ }^{13}$ Simon H. (voir note précédemment).
} 
parties prenantes du projet présents dans cet environnement. L'entrepreneur est le catalyseur de ces échanges avec l'environnement. II est fortement conseillé aux porteurs de projet de ne pas rester isolés dans leur représentation et leur façon de penser. L'interaction avec les parties prenantes favorise l'enrichissement du scénario initial. Le facilitateur apparaît comme important dans le dispositif d'accompagnement entrepreneurial dans la mesure où il va permettre à l'entrepreneur de prendre conscience des interactions avec l'environnement et de les organiser. Le scénario se construit donc en interaction avec les parties prenantes en lien avec le projet. L'entrepreneur se retrouve finalement en situation de co-conception. Apparaît alors la nécessité de pouvoir traduire ce scénario auprès des parties prenantes du projet, de définir une représentation partageable pour tous. L'usage du préfixe « co »permet d'insister sur l'aspect dynamique de la conception du scénario. Le facilitateur apparaît comme celui qui va permettre de rendre partageable le scénario envisagé par l'entrepreneur. II est aussi celui qui amène à prendre conscience de la nécessité de traduire ce scénario. L'accompagnateur doit faire preuve de cette forme d'intelligence qui consiste moins en la résolution d'un problème qu'en la mise en forme d'un monde partageable. La traduction devrait permettre aux différentes parties prenantes de parvenir à se comprendre en vue de travailler ensemble. Le scénario devient donc un instrument de dialogue entre le porteur de projet et les différentes parties prenantes du projet. La traduction est donc un enjeu important car le scénario du porteur de projet n'est pas transposable tel quel. Elle nécessite bien souvent de passer d'un niveau implicite à un niveau explicite. Dominika, ayant exprimé son scénario, elle a réussi à identifier les points d'incohérence de son projet. Le facilitateur l'a aidée à traduire son projet par rapport à des parties prenantes qui ont des attentes différentes, voire des fois divergentes. En allant sur le terrain, il 
s'agit alors pour Dominika de valider ou d'invalider son scénario. Cette démarche lui a permis de gagner beaucoup de temps au lieu de devoir faire une étude de marché pour en comprendre le fonctionnement. Dans cette logique, un lien peut être fait de façon générale avec les inférences bayésiennes ${ }^{14}$ ou, de façon plus ciblée sur l'entrepreneuriat, avec le Lean Startup.

La traduction ne va pas sans difficultés. Aussi est-il nécessaire de favoriser le dialogue entre les différentes parties prenantes. Là encore, le facilitateur peut jouer un rôle d'intermédiation. Cela passe par le développement d'un langage commun autour du scénario en construction. Or, la difficulté actuelle repose sur la création et le développement de ce langage, les différentes parties prenantes parlant souvent des langages « différents ». Ces situations requièrent ainsi une personne maîtrisant ces différents langages. Celle-ci peut être le facilitateur car, en tant qu'accompagnateur dans le domaine de l'entrepreneuriat, il a une connaissance des différentes parties prenantes en lien avec le projet de l'entrepreneur. Ce facilitateur doit être envisagé comme un acteur de la cohérence entre le projet de l'entrepreneur et le scénario développé. Son rôle est donc multiple. En effet, il doit favoriser :

- l'émergence d'un langage commun ;

- le développement et l'enrichissement de ce langage ;

- et sa diffusion.

Cet article a abordé la notion d'accompagnement sous une posture différente : la posture du facilitateur. Celle-ci n'est pas en contradiction avec la posture habituelle

\footnotetext{
${ }^{14}$ Démarche consistant à formuler une solution a priori à partir de son expérience préalable et de mettre en place des actions pour valider ou non cette solution.
} 
prise en matière d'accompagnement entrepreneurial. Bien au contraire, le facilitateur vient enrichir les activités qu'on retrouve habituellement au niveau de l'accompagnement entrepreneurial, notamment autour du plan d'affaires. Cette posture s'appuie sur les avancées que l'on peut retrouver plus largement dans le domaine de l'accompagnement personnel. Au niveau de l'entrepreneuriat, la posture du facilitateur permet non seulement à l'entrepreneur une meilleure compréhension de son propre projet, mais aussi de développer la robustesse de celui-ci, notamment à l'égard des parties prenantes du projet. La posture du facilitateur est particulièrement intéressante au début de la rencontre entre l'entrepreneur et l'accompagnateur. L'exemple présenté en fil rouge de cet article à travers le cas de Dominika, jeune entrepreneure dans le domaine de l'import-export, permet de comprendre, entre autres, l'intérêt de travailler très tôt avec des porteurs de projet. Aujourd'hui, l'entreprise de Dominika a un an à son actif. Elle vient de recruter son premier salarié et elle envisage le développement sur de nouveaux marchés. Toutefois, la posture du facilitateur est loin d'être naturelle dans un environnement largement formaté et conditionné à la posture du réparateur. II conviendrait donc d'avancer sur cette posture, notamment en y formant les accompagnateurs.

Nous avons cherché à travers cet article à présenter dans un premier temps les deux postures que sont celles du réparateur et du facilitateur. L'objectif en était, à travers un tableau synthétisant ces postures, de montrer tout particulièrement la différence entre les deux. Dans un second temps, nous sommes revenus plus en détail sur les apports et les enjeux de la posture du facilitateur et, notamment, sur son rôle dans la conception et la traduction du scénario envisagé. Trop souvent négligée, cette posture reste encore mal comprise car elle se positionne essentiellement sur des aspects moins rationnels. Au final, en matière d'accompagnement entrepreneurial, 
nous nous retrouvons tel l'ivrogne qui ne cherche ses clefs perdues que sous le lampadaire, car c'est le seul endroit où il y ait de la lumière. II est nécessaire de changer notre façon de voir les choses et donc de faire évoluer la posture en matière d'accompagnement entrepreneurial vers celle du facilitateur en complément de celle, plus classique, du réparateur. Au final, cela permet de renfoncer l'intérêt et la légitimité de l'accompagnement entrepreneurial. Si beaucoup d'entreprises se créent en dehors du giron des structures de l'accompagnement entrepreneurial, il conviendrait, non de remettre en cause le rôle de ces structures mais plutôt de les amener à compléter leur posture qui reste encore essentiellement centrée sur la réparation et le plan d'affaires. Ces réflexions devraient nous inciter à nous interroger sur le métier de l'accompagnement et à faire évoluer les pratiques du domaine. Cela ne saurait se faire sans l'appui des financeurs politiques qui doivent aussi faire évoluer leurs représentations de l'accompagnement. Car, l'accompagnement et son financement restent essentiellement publics en France. 